\begin{tabular}{|c|l|}
\hline Title & Fabrication and low-temperature transport properties of selectively grown dual-gated single electron transistors \\
\hline Author(s) & Motohisa, J.; Nakajima, F.; Fukui, T.; van der Wiel, W. G.; Elzerman, J. M.; De Franceschi, S.; Kouwenhoven, L. P. \\
\hline Citation & $\begin{array}{l}\text { A pplied Physics Letters, 80(15), 2797-2799 } \\
\text { https://doi.org/10.1063/1470246 }\end{array}$ \\
\hline Issue Date & $2002-0415$ \\
\hline Doc URL & http://hdl.handle.net/2115/5522 \\
\hline Rights & Copyright $\odot 2002$ A merican Institute of Physics \\
\hline Type & article \\
\hline File Information & APL80-15.pdf \\
\hline
\end{tabular}

Instructions for use 


\title{
Fabrication and low-temperature transport properties of selectively grown dual-gated single-electron transistors
}

\author{
J. Motohisa, ${ }^{a}$ F. Nakajima, and T. Fukui \\ Research Center for Integrated Quantum Electronics, Hokkaido University, North 13 West 8, \\ Sapporo 060-8628, Japan \\ W. G. van der Wiel, J. M. Elzerman, and S. De Franceschi, and L. P. Kouwenhoven \\ Department of Applied Physics and DIMES, Delft University of Technology, P. O. Box 5046, 2600 GA Delft, \\ The Netherlands
}

(Received 14 January 2002; accepted for publicztion 25 February 2002)

\begin{abstract}
We report on the fabrication of a dual-gated single-electron transistor (SET) based on a quantum dot (QD) formed by selective area growth of metalorganic vapor-phase epitaxy, and its low-temperature transport properties. We observe clear Coulomb oscillations in a SET fabricated in combination with direct growth of nanostructures and lithographically defined metal gates. The magnetic field dependence of the Coulomb oscillations as well as the Coulomb diamonds suggest strong carrier confinement in our QD. (C) 2002 American Institute of Physics. [DOI: 10.1063/1.1470246]
\end{abstract}

Single-electron transport through semiconductor quantum dots (QDs) has recently attracted considerable attention. From the application point of view, single-electron devices utilizing Coulomb blockade effects have the potential to realize circuits with high-density integration and low power consumption. ${ }^{1}$ From the physics point of view, the threedimensional confinement in a QD gives rise to a discrete energy spectrum in analogy to atoms. The shell structure can be detected by single-electron transport. ${ }^{2}$ Furthermore, when the QD has a net spin, it can be considered as a localized magnetic impurity with tunable parameters, offering a playground for tunable Kondo physics. ${ }^{3}$ Realization of very small QDs is required for possible operation at high temperatures.

We have reported before on the fabrication of singleelectron devices and circuits by using selective area metalorganic vapor-phase epitaxy (SA-MOVPE) on partially masked substrates. ${ }^{4-6}$ SA-MOVPE technology allows us to fabricate various kinds of semiconductor quantum nanostructures and their dense arrays only with one-step growth by using appropriate mask designing of the substrate. Therefore, it is possible to realize integrated circuits based on nanostructures and single-electron devices. ${ }^{5}$ In particular, we have recently reported on quantum dot arrays and dot-wire coupled structures by utilizing a zig-zag mask pattern. ${ }^{7} \mathrm{We}$ also have proposed single-electron transistors (SETs) having much smaller QDs and stronger lateral confinement as compared to our conventional structures, and SET operation has partially been demonstrated.

So far, our study of the transport properties of SETs fabricated by SA-MOVPE was limited to a narrow temperature region and Coulomb oscillations were observed only in a limited range of gate and source-drain voltages. In this letter, we extend our investigation to low temperature measurements. The implementation of dual-gated structures enhance the tunability of the potential of the QD and tunneling barriers. We have demonstrated clear Coulomb oscillations

\footnotetext{
a) Author to whom correspondence should be addressed; electronic mail: motohisa@rciqe.hokudai.ac.jp
}

in a SET fabricated in combination with crystal growth and dual gates defined by lithographic techniques. Our devices have shown the possibility to explore single-electron transport and the Kondo effect, owing to strong confinement.

The secondary electron microscopy (SEM) image of the present QD device is shown in Fig. 1(a). For its fabrication, we first prepare a masked substrate of Fig. 1(b). Starting with a SiON-coated GaAs (001) substrate, $\mathrm{SiON}$ in the zig-zag shaped area is partly removed by electron-beam lithography wet chemical etching. The edges of the opening are either along the [110] or [010] directions. Next, a $n$-AlGaAs/GaAs/AlGaAs selectively doped double heterostructure is grown by MOVPE on an opening area where the (001) GaAs surface is exposed. Because of the evolution of $\{111\} \mathrm{B}$ and $\{110\}$ facet sidewalls during the growth, we have a width modulated channel of the two-dimensional electron gas (2DEG) in the GaAs layer following the mask pattern, as shown in Fig. 1(c). As a result, a small, diamond-shaped 2DEG region is formed in between two constrictions. Details of the growth process are reported elsewhere. ${ }^{7}$ Then, we define two gates, a top gate and a side gate, near this region, as shown in the SEM image. The QD is formed between the

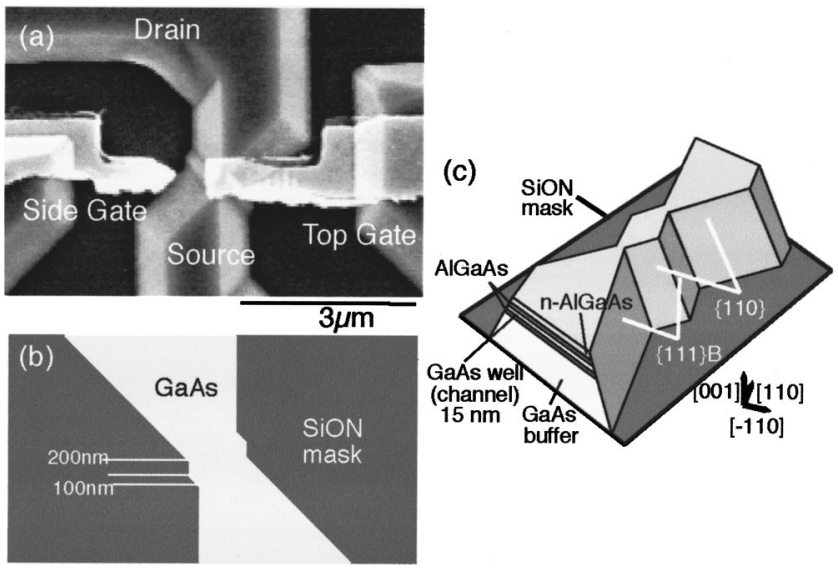

FIG. 1. (a) SEM image of the device. Schematic illustration of (b) the mask pattern and (c) layer structure. 
two constrictions when a negative top gate voltage is applied. The big reservoirs on both sides of the QD correspond to the source and drain contacts.

In this device, the top gate changes both the electrostatic potential of the QD and the tunneling barrier characteristics. ${ }^{7}$ On the other hand, the side gate mainly effects the electrostatic potential of the dot, although some effect on the barriers and confinement is unavoidable. Thus, the present dualgated structure allows one to control the electrostatic potential of the QD nearly independently of the tunneling resistance and the dot shape. Hence, we can investigate single-electron transport in the case of weak and strong coupling of the QD to its leads. Since the channel lays about $50 \mathrm{~nm}$ beneath the surface, we estimate the geometrical size of the dot to be $320 \mathrm{~nm}$, which is slightly larger than the devices reported previously. ${ }^{7}$ The QD becomes effectively smaller by the application of a negative top gate voltage. The electron concentration and mobility of 2DEG formed on the unmasked reference substrate is $8.5 \times 10^{11} \mathrm{~cm}^{-2}$ and 6.6 $\times 10^{4} \mathrm{~cm}^{2} / \mathrm{V} \mathrm{s}$, respectively, at $77 \mathrm{~K}$. The sample is measured in a dilution refrigerator with a base temperature of $15 \mathrm{mK}$. We measure the source-drain conductance, $G$, of the device with a constant dc bias of $10 \mu \mathrm{V}$, or the differential conductance $d I_{\mathrm{DS}} / d V_{\mathrm{DS}}$ using a lock-in technique with an ac bias of $5 \mu \mathrm{V}$ superimposed on the dc bias, $V_{\mathrm{DS}}$.

Without applying a negative top gate voltage, $V_{\mathrm{TG}}$, the conductance through the QD hardly changes when sweeping the side gate voltage, $V_{\mathrm{SG}}$. However, by applying a voltage more negative than about $-250 \mathrm{mV}$ to $V_{\mathrm{TG}}$, the conductance shows Coulomb oscillations as a function of $V_{\mathrm{SG}}$. Figure 2(a) shows typical Coulomb oscillations for different top gate voltages. At the same time, the peak of the oscillations shifts to more negative side gate values when $V_{\mathrm{TG}}$ is made more positive. Similar Coulomb oscillations for $V_{\mathrm{SG}}$ and their shift with respect to $V_{\mathrm{TG}}$ are observed for $-265 \mathrm{mV}$ $<V_{\mathrm{TG}}<-285 \mathrm{mV}$, as shown in Fig. 2(b). For these top gate voltages, the valley conductance as well as the Coulomb peaks seem to be enhanced, as the tunneling resistance of the barrier is reduced as compared to the case of more negative $V_{\mathrm{TG}}$. These results clearly indicate that a $\mathrm{QD}$ is formed in our device by applying a sufficiently negative voltage to the top gate. We note that, in Fig. 2(b), a plateau-like structure is observed as indicated by the arrow. We will discuss its origin later. We also note that the peak height also changes with $V_{\mathrm{TG}}$ in a nonmonotonic manner. We think that by changing $V_{\mathrm{TG}}$, different wave functions may be involved in transport, resulting in a different tunnel probability.

Figure 3 shows a gray-scale plot of the differential conductance, $d I_{\mathrm{DS}} / d V_{\mathrm{DS}}$, for $V_{\mathrm{TG}}=-299 \mathrm{mV}$ [thicker trace in Fig. 2(a)]. We observe clear Coulomb diamonds. As one can see in Fig. 3, the Coulomb gap $U_{\mathrm{C}}$ strongly depends on the side gate voltage $V_{\mathrm{SG}}$. The maximum and minimum values for $U_{\mathrm{C}}$ are $3.30 \mathrm{mV}$ and $1.34 \mathrm{mV}$, respectively. One of the possible explanations for such a dependence would be the change of the effective dot size by the side gate bias. This would partly explain the results, because the alpha factor ${ }^{8}$ ( $\alpha=C_{\mathrm{G}} / C_{\mathrm{dot}}$, where $C_{\mathrm{G}}$ the capacitance between dot and the side gate, $C_{\mathrm{dot}}$ the total capacitance of the dot) increases monotonically when $V_{\mathrm{SG}}$ is decreased and $C_{\mathrm{G}}$ would be more sensitive to $C_{\mathrm{dot}}$ for $V_{\mathrm{SG}}$. However, the Coulomb gap does Downloaded 23 Jan 2006 to 133.87 .26 .100 . Redistribution subject
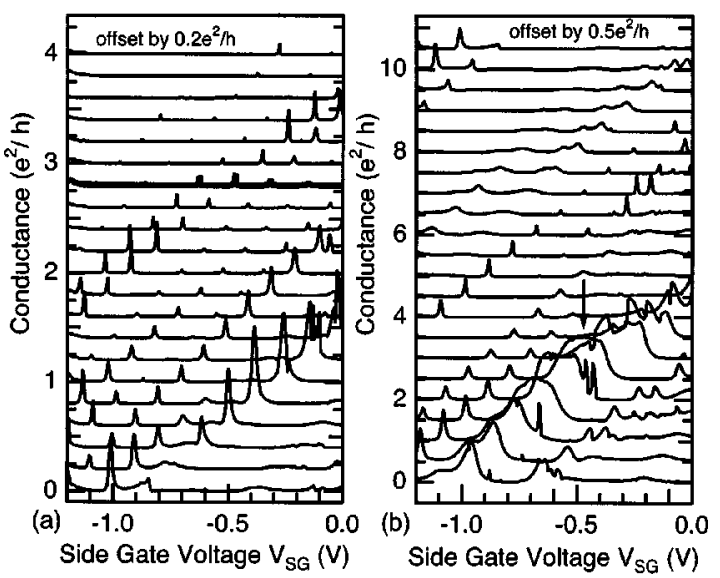

FIG. 2. Linear conductance of the device measured at base temperature ( $\sim 15 \mathrm{mK}$ ), for fixed top gate voltage, $V_{\mathrm{TG}} \cdot V_{\mathrm{TG}}$ is varied (a) from $-305 \mathrm{mV}$ (top) to $-285 \mathrm{mV}$ (bottom), and (b) from $-285 \mathrm{mV}$ (top) to $-265 \mathrm{mV}$ (bottom). The step size for $V_{\mathrm{TG}}$ is $1 \mathrm{mV}$ for each figure.

not show monotonic change for $V_{\mathrm{SG}}$. The second possibility would be the dependence of the addition energy on the number $N$ of electrons in the QD. This can happen, for example, when the QD has a shell structure similar to real atoms ( or artificial atoms). ${ }^{2}$ We will come back to this point later.

We also measure the evolution of the Coulomb oscillations with magnetic field. The results are shown in Fig. 4 in a gray-scale plot of conductance, $G$, as a function of magnetic field $B$, and side gate voltage $V_{\mathrm{SG}}$. Here, $V_{\mathrm{TG}}$ is fixed at $-299 \mathrm{mV}$, and the magnetic field is applied normal to the surface. Although the overall behavior is somewhat complicated, the Coulomb peaks show a parabolic shift for $0<B$ $<2 \mathrm{~T}$, particularly for the peaks observed at more negative $V_{\mathrm{SG}}$. In addition, we also can see some kinks in the peak shift, which is probably due to the crossing of ground states. In some ranges of magnetic field and side gate voltage, the



FIG. 3. Gray-scale plot of the differential conductance $d I_{\mathrm{SD}} / V_{\mathrm{SD}}$, plotted as a function of both $V_{\mathrm{SD}}$ and $V_{\mathrm{SG}}$. The top gate voltage is $-299 \mathrm{mV}$. For

$V_{\mathrm{SG}}<0.7 \mathrm{~V}$, the contrast is enhanced by factor of 5 . 


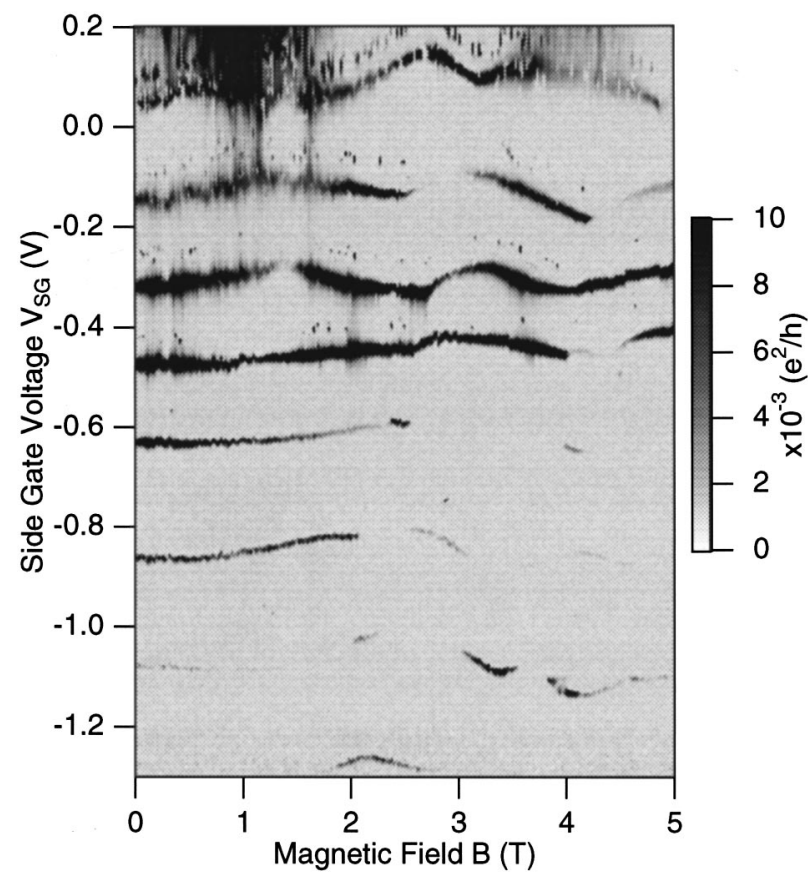

FIG. 4. Magnetic field dependence of the Coulomb oscillation with respect to the side gate voltage $V_{\mathrm{SG}}$. The top gate voltage is $-299 \mathrm{mV}$.

peaks are suppressed considerably. On the other hand, peaks reappear around $1.8<B<2.8 \mathrm{~T}$, for $V_{\mathrm{SG}} \sim-1.25 \mathrm{~V}$. Such behavior of the peaks could suggest that there is an overlap of the wave functions in the QD which leads to changes by the magnetic field and gate voltages in a rather complicated fashion. However, these characteristics are not clearly understood yet.

From the results of Figs. 3 and 4, we estimate the peak shift by the magnetic field at $2 \mathrm{~T}$ is order of $0.12 \mathrm{meV}$. This small shift of the Coulomb peaks for $0<B<2 \mathrm{~T}$ strongly suggests that the carrier confinement in our QD is strong and the number $N$ of electrons in a QD is very small. The similarity of the magnetic field dependence between our present results and the results of Tarucha et al. ${ }^{2}$ also forms evidence for strong confinement of a small number of electrons and atomic-like properties in our QD. On the other hand, the level separation estimated from the excited state spectrum, is very small $(0.18-0.30 \mathrm{meV})$ and does not imply strong quantum confinement. It is difficult to obtain an estimate for $N$ derived from the dot diameter, $d$ (estimated from the maximum charging energy), and the electron density.

Now, we would like to discuss the origin of the plateaulike structure indicated by the arrow in Fig. 2(b). We think this broad peak originates from the Kondo effect, which is observed when the QD has a net spin (usually, $S=1 / 2$ ). ${ }^{3}$ Although we have not measured temperature dependence, systematic measurements, ${ }^{10}$ such as differential conductance, $d I_{\mathrm{DS}} / d V_{\mathrm{DS}}$ where we have observed a characteristic peak at $V_{\mathrm{DS}}=0$, strongly suggests the existence of the Kondo effects close to the unitary limit. ${ }^{11}$ Furthermore, our study on higher magnetic field $(\sim 4.6 \mathrm{~T})$ clearly shows the existence of the Kondo effect in the similar top gate voltage conditions. A detailed study of the Kondo effect at high magnetic field will be presented elsewhere. ${ }^{12}$
Finally, we describe some of the technological impact of our present device structures. First of all, direct formation methods of nanostructures utilizing epitaxial growth are very effective to realize structures or devices with very small high-density QDs, which we showed in our previous papers. Dilger et al. also have reported low-temperature (down to $22 \mathrm{mK}$ ) Coulomb oscillations in a QD device formed by direct molecular-beam epitaxial growth on patterned GaAs substrates. ${ }^{9}$ In these devices, the size of the QDs is estimated to be about or less than $150 \mathrm{~nm}$. Secondly, the dual-gated structure enhances the tuning range to observe Coulomb blockade in such epitaxially grown structures. In our previous devices, the gate bias conditions to observe Coulomb oscillations are very limited and are very close to their threshold voltage. In the case of the device of Dilger et al., in which an in-plane gate fabricated during the growth is used to tune the electrostatic potential of the QD, a gradual increase of the valley conductance of the Coulomb oscillations is observed. This indicates a dependence of the tunneling resistance between the QD and the leads on the in-plane gate bias. Therefore, the dual-gated structure forms an appealing alternative to enhance the tunability of the QD device. Such gate structure is also advantageous for the application of integrated circuits, such as complementary-type single-electron inverters, ${ }^{13}$ in which control of the peaks of Coulomb oscillation is required.

The authors acknowledge Professors H. Hasegawa, H. Akera, M. Eto, and S. Kasai for fruitful discussions. This work is partly financially supported by a Grant-in-Aid for Scientific Research, supported by Ministry of Education, Culture, Sports, Science, and Technology, Japan.

${ }^{1}$ K. K. Likharev, IEEE Trans. Magn. 23, 1142 (1987).

${ }^{2}$ S. Tarucha, D. G. Austing, T. Honda, R. J. van der Hage, and L. P. Kouwenhoven, Phys. Rev. Lett. 77, 3613 (1996).

${ }^{3}$ D. Goldhaber-Gordon, H. Shtrikman, D. Mahalu, D. Abusch-Magder, U. Meirav, and M. A. Kastner, Nature (London) 391, 156 (1998); S. M. Cronenwett, T. H. Oosterkamp, and L. P. Kouwenhoven, Science 281, 540 (1998); J. Schmid, J. Weiss, K. Eberl, and K. von Klitzing, Physica B 256, 182 (1998).

${ }^{4}$ K. Kumakura, J. Motohisa, and T. Fukui, Solid-State Electron. 42, 1227 (1998); K. Kumakura, J. Motohisa, and T. Fukui, Physica E (Amsterdam) 2, 809 (1998).

${ }^{5}$ F. Nakajima, K. Kumakura, J. Motohisa, and T. Fukui, Jpn. J. Appl. Phys., Part 1 38, 415 (1999)

${ }^{6}$ F. Nakajima, J. Motohisa, and T. Fukui, Appl. Surf. Sci. 162, 650 (2000).

${ }^{7}$ F. Nakajima, Y. Ogasawara, J. Motohisa, and T. Fukui, J. Appl. Phys. 90, 2606 (2001).

${ }^{8}$ J. Weis, R. J. Haug, K. von Klitzing, and K. Ploog, Phys. Rev. Lett. 71, 4019 (1993).

${ }^{9}$ M. Digler, R. J. Haug, K. Eberl, and K. von Klitzing, Semicond. Sci. Technol. 11, 1493 (1996).

${ }^{10}$ J. Motohisa, W. G. van der Wiel, J. M. Elzerman, S. De Franceschi, F. Nakajima, Y. Ogasawara, T. Fukui, and L. P. Kouwenhoven, Physica E (to be published).

${ }^{11}$ W. G. van der Wiel, S. De Franceschi, T. Fujisawa, J. M. Elzerman, S. Tarucha, and L. P. Kouwenhoven, Science 289, 2105 (2000).

${ }^{12}$ W. G. van der Wiel, S. De Franceschi, J. M. Elzerman, S. Tarucha, L. P. Kouwenhoven, J. Motohisa, F. Nakajima, and T. Fukui, Phys. Rev. Lett. (to be published).

${ }^{13}$ J. R. Tucker, J. Appl. Phys. 72, 4399 (1992). 